\title{
Representação Gráfica de Tecidos Orgânicos Humanos Vivos
}

\author{
Augusto Luengo Pereira Nunes \\ Instituto de Informática \\ Universidade Federal do Rio Grande do Sul \\ Porto Alegre, Rio Grande do Sul, Brasil.
}

\author{
Marcelo Walter \\ Instituto de Informática \\ Universidade Federal do Rio Grande do Sul \\ Porto Alegre, Rio Grande do Sul, Brasil
}

\author{
Anderson Maciel \\ Instituto de Informática \\ Universidade Federal do Rio Grande do Sul \\ Porto Alegre, Rio Grande do Sul, Brasil
}

\begin{abstract}
The medical applications like surgery virtual simulators are important study and training tools for Medicine. The virtual scenes visual quality improves the relevant skills development, according to the PSVR (Patient Specific Virtual Reality) simulation. Moreover, organic tissue graphics representation techniques are essential to the realism of such simulations. The Computer Graphics presents particular approaches which we can classify according to the simulation process of the light and organic material interaction. This work presents the major contributions to the human tissue simulation, mainly from internal organs, and proposes a new taxonomy to such methods.
\end{abstract}

Index Terms - Virtual Surgery, Organic Tissue Simulation, Visual Realism

Resumo - Aplicações médicas como simuladores virtuais de cirurgia são importantes ferramentas de estudo e treinamento para a área médica. A qualidade visual das cenas virtuais beneficia o desenvolvimento de habilidades pertinentes, de acordo com o conceito de simulação PSVR (Patient Specific Virtual Reality). Neste contexto, técnicas de representação gráfica de tecidos orgânicos são fundamentais para o realismo de tais simulações. A Computação Gráfica apresenta abordagens características, que podem ser classificadas de acordo com o processo de simulação da interação entre a luz e o material orgânico. $O$ presente trabalho apresenta as principais contribuições para a simulação de tecido humano vivo, principalmente de órgãos internos, e propõe uma nova taxonomia para tais métodos.

Palavras-chave - Cirurgia Virtual, Simulação de Tecidos Orgânicos, Realismo Visual.

\section{I.INTRODUÇÃO}

A descrição de materiais como componentes para processos de síntese de imagens é uma das principais atividades da Computação Gráfica (CG). Tais simulações podem envolver desde aproximações pautadas apenas por julgamentos visuais até modelagens dos processos físicos presentes na interação luz-matéria [1].

As particularidades de um material, assim como sua microestrutura, podem ser estudadas através de dispositivos espectrais [2] e representadas por modelos com poder de expres- são compatível [3]. Alguns materiais possuem características restritivas para a análise de suas propriedades ópticas. Neste contexto, os tecidos orgânicos humanos representam uma das classes mais desafiadoras [4]. Naturalmente, tecidos in vivo possuem aspecto visual dinâmico, governado por fatores associados ao histórico médico (ou anamnese) de cada indiví- duo, e pode variar drasticamente no período post mortem [5].

A simulação de cirurgias é a principal aplicação para a modelagem de tecidos orgânicos humanos, determinante no treinamento de novos cirurgiões quanto a transferência de conhecimento [6]. Além disso, tal aplicação tem como meta reproduzir todo ambiente interno do corpo humano, levando em conta não só fatores anatômicos e mecânicos, mas também de aspecto visual, como a transparência e heterogeneidade de tecidos orgânicos [7].

O presente trabalho apresenta o estado da arte de técnicas para a representação de tecidos do corpo humano na área da Computação Gráfica. Além disso, uma nova taxonomia para classes de tais técnicas é proposta com base nos processos geradores das imagens e inspirada nas definições de realismo de Ferwerda [1].

\section{TAXONOMIA PARA REPRESENTAÇÃO DE TECIDOS ORGÂNICOS}

O Fotorrealismo de imagens sintéticas pode ser definido como sua capacidade de produzir respostas no sistema visual humano como as de imagens reais, tornando-as indistinguíveis [1]. Neste caso, o processo de rendering pode ser completamente arbitrário, sem considerar processos físicos envolvidos na interação luz-matéria. Tais técnicas tendem a envolver, em alguma de suas etapas, intervenções manuais ou artísticas, como composição de texturas e correção de suas descontinuidades [8], [9]. No contexto de tecidos orgânicos, podemos reunir nesta classe os trabalhos que baseiam sua metodologia na aproximação do aspecto orgânico com o uso de texturas geradas por funções de ruído [10], [11], junto a equações de iluminação baseadas no modelo de Phong [12]. A amostragem de características radiométricas de tecidos orgânicos caracteriza métodos de Realismo Físico, que buscam primeiro obter a resposta de um tipo de material quando iluminado, para então orientar modelos de iluminação clássicos de acordo com suas medições [13]. O dinamismo de tecidos orgânicos requer que as amostragens sejam feitas in vivo, o que representa um grande impeditivo para a popularização dessa classe de técnicas, pois mesmo na área médica, para alguns tipos de tecidos, obter amostras de interação luz- matéria nestas condições é pouco convencional [5]. Além disso, cada amostragem de um tecido em particular, pertence a um indivíduo, o que penaliza a variabilidade da customização de simulações. 
Finalmente, técnicas que buscam produzir imagens sintéticas biologicamente plausíveis através das propriedades ópticas de componentes orgânicos do tecido, podem ser descritas como baseadas em Princípios Fundamentais (FirstPrinciples) [14].

Um único modelo desta classe para um órgão em particular, pode produzir diversos aspectos visuais diferentes, respeitando as características do tecido simulado. Utilizando a literatura disponível na área médica, e até mesmo medições e modelos matemáticos preditivos, um número cres- cente de trabalhos vem adaptando tais informações para o uso em processos de rendering de tecido orgânico em CG [15]- [18]. A Figura 1 ilustra o processo de síntese de imagens de acordo com as classes apresentadas.

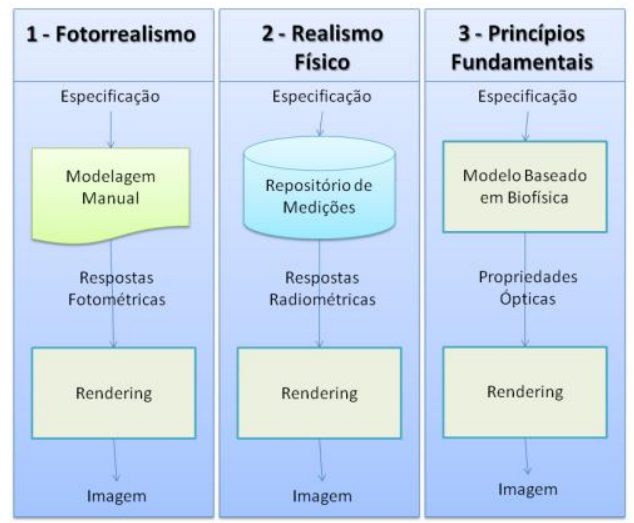

Figura 1. Taxonomia para tipos de modelagem da Computação Gráfica para a interação luz-matéria de tecidos orgânicos.

\section{A. Fotorrealismo}

O trabalho de Sunguroff e Greenberg [19] introduziu visualização tridimensional de estruturas orgânicas em seu sistema para planejamento do tratamento de tumores no cérebro, com base no uso de Gouraud shading [20] e no modelo de iluminação de Blinn [21]. Como uma forma de aumentar a fidelidade da síntese de imagens em aplicações médicas, Barillot et al. [22] procuraram representar estruturas finas (vasos sanguíneos do cérebro humano) com o auxílio de equipamentos médicos mais precisos.

Como uma solução para enriquecer uma cena tridimensional sem aumentar a quantidade de geometria processada para gerar a imagem final, Catmull [23] introduziu o Mapeamento de Textura. Esta técnica proporciona o mapeamento de imagens (fotografias, por exemplo) sobre a superfície de objetos virtuais. A continuidade das amostras de textura e a supressão de distorções provocadas pelo mapeamento das mesmas em objetos tridimensionais são abordados por Neyret e Cani [8] em sua simulação da superfície do fígado. Tal trabalho su- gere um algoritmo para síntese de textura procedural e uma solução para o mapeamento coerente do resultado. Notando o comportamento especular do fígado quando vivo, Neyret et al. [24] adicionam ao resultado anterior, uma simplificação do Environment Mapping [25] para obter a reflexão da fonte de luz de um simulador de Laparoscopia, representando o aspecto molhado dos órgãos humanos.

O modelo de iluminação local utilizado por Neyret é o de Phong [12], baseado em observações empíricas da interação luz-matéria. A avaliação da equação de iluminação é feita a cada fragmento de imagem, através de Phong Shading. Elhelw et al. [9] argumentam que tais técnicas, utilizadas em conjunto com superfícies lisas, produzem um efeito semelhante a materiais plásticos, o que diverge do objetivo principal de simulação de estruturas orgânicas. Tais autores propõem o uso de Bump Mapping [26] gerado por função de ruído, para representar níveis de rugosidade de tecidos orgânicos. Além disso, utilizam um mapa de refrações buscando simular a transparência desse tipo de material.

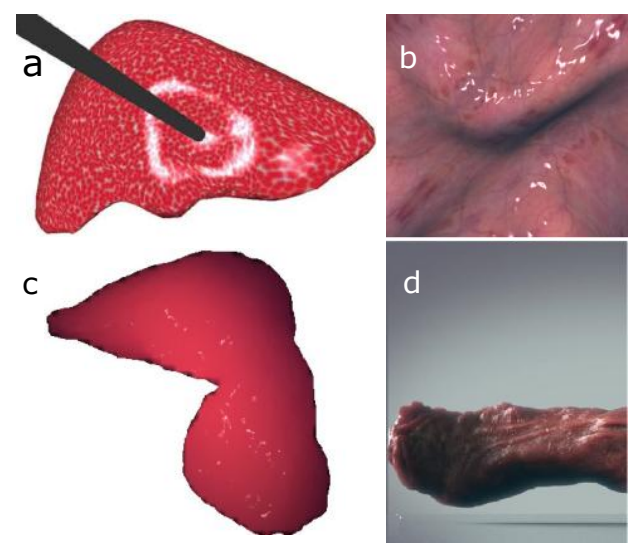

Figura 2. a - Redering de um fígado humano através da simulação de seu padrão de textura e camada especular [24]. b Simulação de tecido orgânico com aspecto 'molhado' [29]. c Simulação de tecido composto por camadas de fluidos orgânicos [27]. d - Estrutura orgânica gerada através de técnicas clássicas da $\mathrm{CG}$, como mapas de textura e perturbações locais na geometria [30].

Superfícies rugosas cobertas por fluidos e compostas por múltiplas camadas, tendem a apresentar subsurface scattering, onde a luz entra e sai por pontos diferentes da superfície. Hao et al. [27] abordam tal característica de tecidos orgânicos, introduzindo o uso do modelo de aproximação para dispersão múltipla de Jensen et al. [28], visando simular a propagação da luz entre camadas de tecidos de um fígado, em conjunto com o modelo de reflexão especular de Blinn-Phong [21] modificado em função de Bump Mapping.

O uso de texturas e outras técnicas clássicas de CG para a aproximação do aspecto visual de tecidos orgânicos proporciona bom fotorrealismo para aplicações médicas, como mostra a Figura 2. Entretanto, o processo de modelagem através da união desses diferentes componentes gráficos, frequentemente requer intervenções manuais. De fato, tal característica penaliza a customização eficiente do rendering. Uma vez produzido um resultado, quando necessário representar o mesmo tecido para um indivíduo diferente, inevitavelmente alguns componentes deverão ser novamente manipulados.

\section{B. Realismo Físico}

Desde a introdução do conceito de BRDF (Bidirectional Reflectance Distribution Function) como um modelo geral para 
a interação luz-matéria, feita por Nicodemus [3], diversos trabalhos propuseram técnicas para medir seus valores para materiais do mundo natural. Além disso, temos evoluções do conceito original de BRDF para representar materiais heterogêneos (SVBRDF - Spatially Varying Bidirectional Reflectance Distribution Function), com camadas translúcidas (BTDF - Bidirectional Transmittance Distribution Function) ou mesoestruturas (BTF - Bidirectional Texture Function). Uma apresentação detalhada de tais conceitos pode ser encontrada no trabalho de Pharr e Humphreys [31]. Tais modelos apresentam resultados que se aproximam do fotorrealismo, mesmo para materiais como a pele do rosto humano [32]. Mas considerando tecidos de órgãos internos do corpo humano, surge um espaço importante sem avanços na mesma proporção.

O estado da arte para este problema está, hoje, na leitura de BRDF.

Um dos métodos mais eficientes para amostragem de BRDF foi apresentado por Marschner [33], baseado no uso de imagens, com o qual foi possível medir características radiométricas da pele humana, além de aproximar os parâmetros do modelo de iluminação de Lafortune [34]. Para materiais de fácil manipulação, tal técnica realiza as amostragens de forma trivial. Mas para ambientes de espaço e tempo limitado, como é o interior do corpo humano, são necessárias técnicas adaptáveis. O trabalho de Chung et al. [13] introduziu a amostragem de BRDF de órgãos internos durante MIS, através de broncoscopias. Seus resultados trazem características radiométricas de brônquios humanos, enquanto vivos. A principal limitação dessa técnica é o fato de permitir apenas amostragens de porções da BRDF onde as direções de entrada e saída de luz são iguais (retro-reflexivas), o que penaliza sua aproximação de parâmetros para modelos de iluminação clássicos [35]. Cenydd et. al. [36] realizaram tentativas de amostragem da BRDF do cérebro humano durante uma cirurgia neurológica aberta através de 5 fotografias obtidas em condições especiais de iluminação. A pouca variabilidade angular da amostragem obtida inviabilizou a recuperação da BRDF, o que levou os autores a optarem por amostrar BRDFs de tecidos orgânicos in vitro. Os dados obtidos foram usados na aproximação dos parâmetros do modelo de Lafortune.

Medições de características radiométricas de materiais orgânicos podem ser usadas em diferentes contextos dentro de aplicações médicas. Yeung et al. [37] apresenta uma técnica para reconstrução de geometrias de órgãos baseada em imagens de MIS, utilizando estimativas de BRDF retro-reflexiva. Algo semelhante é apresentado por Malti e Bartoli [38], porém os valores de BRDF medidos são utilizados na estimativa de parâmetros para o modelo de iluminação de Cook-Torrance [39]. Ambos os casos apenas amostram valores da porção retroreflexiva da BRDF para uso como equações de iluminação para SfS (Shape from Shading) [40].

Técnicas eficientes de amostragem tem sido propostas para diferentes tipos de materiais, como os que apresentam texturas e mesoestruturas [41]. Gradativamente, tal processo vem sendo simplificado, dispensando equipamentos de precisão para posicionamentos de fontes de luz ou câmeras [42]. Além disso, alguns trabalhos buscam diminuir a redundância dos dados obtidos [43]. Entretanto, a leitura completa da BRDF de tecidos de órgãos iternos do corpo humano ainda representa um problema em aberto para a área da Computação Gráfica. A Figura 3 mostra alguns resultados de trabalhos mencionados nesta seção.

\section{Princípios Fundamentais}

Esta seção apresenta trabalhos que consideram a interação luz-matéria dos componentes orgânicos de um tecido para definir modelos de simulação de transporte de luz [14]. A literatura da área biomédica apresenta razoável número de trabalhos dedicados a estudar a óptica de tecidos orgânicos, cujos conceitos fundamentais podem ser apreciados no trabalho de Welch e Gemert [44], enquanto Jacques [5] descreve as propriedades ópticas de diferentes materiais orgânicos.

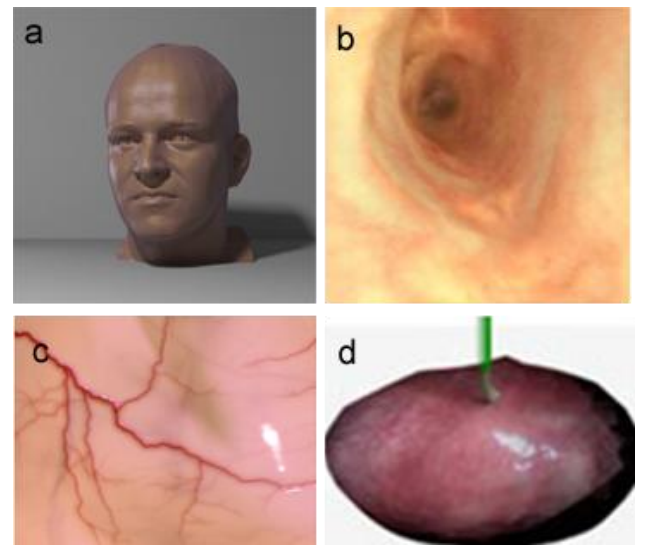

Figura 3. a - Rendering de uma face com BRDF medida pela técnica de Marschner [33]. b - Simulação de broncoscopia com aspecto visual governado pela leitura da BRDF durante uma MIS [13]. c - Rendering da superfície do cérebro humano gerado a partir de uma BRDF orgânica coletada da amostra de um cordeiro [36]. d Reconstrução da geometria de um útero a partir da BRDF medida por Malti e Bartoli [40]

O modelo de Hanrahan e Krueger [45] introduz uma alternativa para simular superfícies compostas por duas camadas através de uma função que atua no caminho óptico da luz dentro de tecidos translúcidos, e obtendo uma aproximação para a solução de primeira ordem das reflexões internas. Tal trabalho considera índices de reflexão, refração e absorção para cada camada do tecido de acordo com medições radiométricas. Os resultados mostram que o modelo proposto pode ser usado para simulação tanto da pele humana, como outros materiais compostos por camadas, como folhas de plantas. So-Ling e Li [46] sugerem uma camada adicional ao modelo anterior para representar a oleosidade da pele humana.

Krishnaswamy e Baranoski [15] apresentam o BioSpec, um modelo para simulação da interação entre a luz e o tecido da pele humana, que simula as respostas radiométricas de seus componentes orgânicos. Modelando o comportamento das camadas derme e epiderme, tais autores produzem simulações não determinísticas (baseado no Método de Monte Carlo MMC) das características reflexivas, de transmissão e absorção de luz pela pele. Para a epiderme, o espalhamento interno da 
luz é calculado com base em dados radiométricos reportados pela área biomédica. Já para a derme, os autores utilizam a Dispersão de Rayleigh, que representa a dispersão da luz por meios com partículas muito menores que o comprimento de onda incidente, de acordo com a Teoria de Mie (que equaciona de forma determinística a interação da luz com meios compostos por partículas) [44]. A absorção de luz é computadacom base na densidade de material pigmentado e seus respectivos índices de absorção, em cada camada. A melanina e $\beta$-caroteno formam os fatores de absorção da epiderme, enquanto o sangue (oxigenado ou desoxigenado), $\beta$ caroteno e bilirrubina formam a derme. $\mathrm{O}$ comportamento do BioSpec foi comparado com dados de medições radiométricas da pele, como as feitas por Marschner [33], mostrando bons resultados.

Chen et al. [18] argumentam que mesmo o BioSpec considera em sua formulação uma distribuição uniforme da orientação, forma e tamanho dos melanossomos (componentes absorcivos da pele), quando faz uso de aproximações de interação luz-matéria, baseadas na Teoria de Mie. Assim, tal modelo é incapaz de simular detour effect (quando a luz interage diversas vezes dentro de organelas das camadas da pele, aumentando seu caminho óptico) [47] ou sieve effect (quando a luz passa diretamente de uma camada para outra) [48]. Tais autores introduzem o HyLIoS (Hyperspectral Light Impingement on Skin) como um modelo para simular interação da luz com as camadas da pele humana, cobrindo o espectro entre $250 \mathrm{~nm}$ e $2500 \mathrm{~nm}$ dos comprimentos de ondas (consideravelmente mais do que a porção visível). Além disso, o modelo proposto apresenta dependência direcional e posicional [3], simulando assim situações nas quais a luz entra e sai da superfície por pontos e ângulos diferentes. A releitura formulada pelo HyLIoS baseia-se da descrição dos aspectos geométricos das partículas orgânicas que compõem a pele, fornecidas pela literatura biomédica, como em Olson et al. [49].

Interpretando o sangue como um tipo de tecido humano com células suspensas em meio a um fluido chamado plasma, Yim et al. [16] descrevem um modelo preditivo para a interação entre luz e tal material. O modelo CLBlood considera em sua formulação propriedades biofísicas das células do san- gue e plasma, além de parâmetros fisiológicos e reológicos (estado do fluido quanto ao transporte de matéria). Além disso, o modelo simula respostas de interações hiper-espectrais para reflexão, absorção e dispersão da luz. Componentes sanguíneos como células vermelhas, células brancas e pla- quetas são modelados segundo as descrições geométricas e resposta espectral reportados pela literatura biomédica. As próprias células vermelhas são decompostas como frações de oxihemoglobina e desoxihemoglobina, para definir o caminho óptico da luz ao atravessar tal estrutura. Como um modelo não determinístico, o aspecto final provido pelo CLBlood é computado através de MMC [17].

Embora os resultados apresentados por trabalhos baseados em Princípios Fundamentais mostrem grande potencial de customização, como ilustra a Figura 4, cada modelo está fortemente ligado ao tecido e órgão específico o qual se dedica a simular. Assim, materiais não abordados pela atual literatura da área, representam problemas em aberto para a Computação Gráfica.

\section{CONCLUSÃO}

O panorama geral de publicações da área de simulação de tecidos, ilustrado na Figura 5, mostra que tal assunto ainda pode receber maior atenção de grupos de pesquisa em CG. De acordo com a taxonomia apresentada por este trabalho, que especifica tais metodologias com base no processo de síntese gerador das imagens, as classes identificadas mostram comportamento concorrente, cujo objetivo é aproximar o aspecto visual da interação entre a luz e tecidos de órgãos vivos.

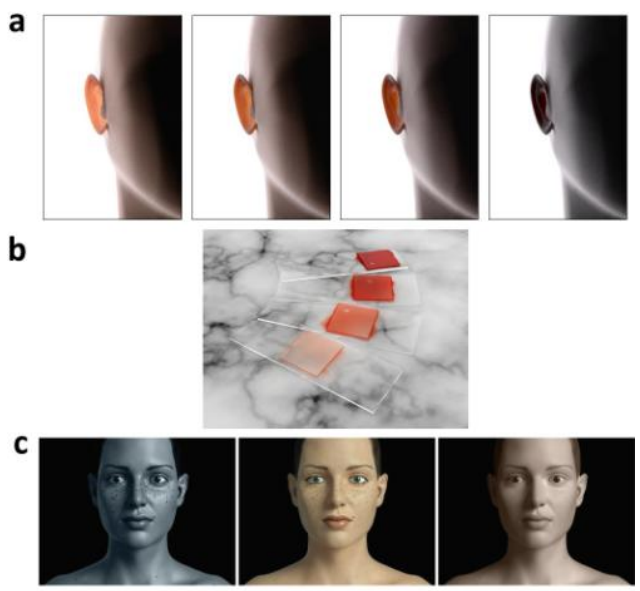

Figura 4. a - Imagens mostrando diferentes resultados do modelo BioSpec em função da variação dos níveis de melanina (da esquerda para a direta, entre $1.9 \%$ até $42 \%$ ) [15]. b - Resultados do CLBlood para diferentes proporções de hematócritos no sangue (de baixo para cima, entre 5\% até 49\%) [17]. c - Simulação do modelo HyLIoS para pele humana sob luz ultravioleta, espectro visível, e luz infravermelha [18]

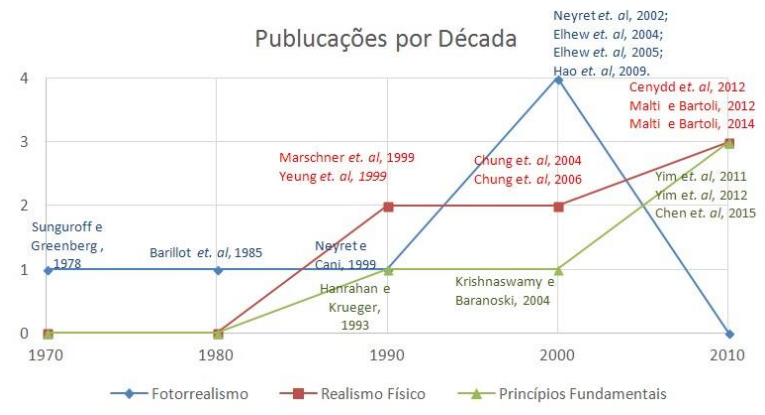

Figura 5. Panorama de contribuições para a representação gráfica de tecidos orgânicos humanos.

Técnicas de Fotorrealismo proporcionam possibilidade de pré-processamento de informações, sendo por isso mais compatíveis com sistemas de tempo real atuais, como simuladores de cirurgia de última geração. Porém, novas contribuições nessa linha de trabalho vem se tornando escassas nos últimos anos. Por outro lado, técnicas de Realismo Físico contam com atenção regular da área, principalmente por apresentar alto grau de customização do rendering de imagens, buscando construir 
uma base de dados para tais materiais a exemplo do Mitsubishi Electric Research Laboratories (MERL) [50]. Além disso, há compatibilidade direta entre dados de amostra- gens de materiais orgânicos e sistemas de rendering baseados em Física [31].

A capacidade de customização do aspecto visual de um tecido de acordo com características de um indivíduo, forma o principal requisito das aplicações médicas da próxima geração, baseadas no paradigma Patient Specific Virtual Reality (PSVR) [51]. Embora de custo computacional alto, métodos baseados em Princípios Fundamentais tem sido propostos em função de sua estrutura parametrizável de acordo com características biofísicas de um indivíduo. Este tipo de técnica evita a recorrente necessidade de amostragens, muitas vezes adaptando protocolos cirúrgicos, comum em métodos de Realismo Físico.

\section{REFERÊNCIAS}

[1] J. A. Ferwerda, "Three varieties of realism in computer graphics," in In Proceedings SPIE Human Vision and Electronic Imaging, 2003, pp. 290-297.

[2] G. J. Ward, "Measuring and modeling anisotropic reflection," SIGGRAPH Comput. Graph., vol. 26, no. 2, pp. 265-272, Jul. 1992. [Online]. Available: http://doi.acm.org/10.1145/142920.134078

[3] F. E. Nicodemus, J. C. Richmond, J. J. Hsia, I. W. Ginsberg, and T. Limperis, "Geometrical considerations and nomenclature for reflectance," in Radiometry, L. B. Wolff, S. A. Shafer, and G. Healey, Eds. USA: Jones and Bartlett Publishers, Inc., 1992, pp. 94-145. [Online]. Available: http://dl.acm.org/citation.cfm?id=136913.136929

[4] J. Dorsey, H. Rushmeier, and F. Sillion, Digital Modeling of Material Appearance. San Francisco, CA, USA: Morgan Kaufmann Publishers Inc., 2008.

[5] S. L. Jacques, "Optical properties of biological tissues: a review," Physics in Medicine and Biology, vol. 58, no. 11, p. R37, 2013. [Online]. Available: http://stacks.iop.org/0031-9155/58/i=11/a=R37

[6] K. S. Lehmann, J. P. Ritz, H. Maass, H. K. Çakmak, U. G. Kuehnapfel,T. Germer, G. Bretthauer, and H. J. Buhr, "A prospective randomized study to test the transfer of basic psychomotor skills from virtual reality to physical reality in a comparable training setting," Annals of Surgery, vol. 241, no. 3, pp. 442-449, 2005. [Online]. Available: http://www.ncbi.nlm.nih.gov/pmc/articles/PMC1356982/

[7] S. Dawson and J. Kaufman, "The imperative for medical simulation," Proceedings of the IEEE, vol. 86, no. 3, pp. 479 -483, mar 1998.

[8] F. Neyret and M.-P. Cani, "Pattern-based texturing revisited," in Proceedings of the 26th annual conference on Computer graphics and interactive techniques, ser. SIGGRAPH '99. New York, NY, USA: ACM Press/Addison-Wesley Publishing Co., 1999, pp. 235-242. [Online]. Available: http://dx.doi.org/10.1145/311535.311561

[9] M. A. Elhelw, B. P. Lo, A. Darzi, and G. zhong Yang, "Real-time photorealistic rendering for surgical simulations with graphics hardware," in in Proc. MIAR 04: Medial Imaging and Augmented Reality, 2004, pp. 346-352.

[10] K. Perlin, "An image synthesizer," SIGGRAPH Comput. Graph., vol. 19, no. 3, pp. 287-296, Jul. 1985. [Online]. Available: http://doi.acm.org/10.1145/325165.325247

[11] S. Worley, "A cellular texture basis function," in Proceedings of the 23rd annual conference on Computer graphics and interactive techniques, ser. SIGGRAPH '96. New York, NY, USA: ACM, 1996, pp. 291-294. [Online]. Available: http://doi.acm.org/10.1145/237170.237267

[12] B. T. Phong, "Illumination for computer generated pictures," Commun. $A C M$, vol. 18, no. 6, pp. 311-317, Jun. 1975. [Online]. Available: http://doi.acm.org/10.1145/360825.360839

[13] A. J. Chung, F. Deligianni, P. Shah, A. Wells, and G. zhong Yang, "Enhancement of visual realism with brdf for patient specific bronchosCopy simulation," in Lecture Notes in Computer Science. Springer VerLag, 2004, pp. 486-493.

[14] G. V. G. Baranoski and A. Krishnaswamy, Light \& Skin Interactions: Simulations for Computer Graphics Applications. San Francisco, CA, USA: Morgan Kaufmann Publishers Inc., 2010.

[15] A. Krishnaswamy and G. V. Baranoski, "A Biophysically-Based
Spectral Model of Light Interaction with Human Skin," Computer GraphicsForum, 2004.

[16] D. Yim, G. V. G. Baranoski, T. F. Chen, B. W. Kimmel, and E. Mir, "On the modeling of light interactions with human blood," 2011 [Online]. Available: https://cs.uwaterloo.ca/research/tr/2011/CS-201130.pdf

[17] D. Yim, G. Baranoski, B. Kimmel, T. Chen, and E. Miranda, "A cellbased light interaction model for human blood," Comput. Graph. Forum, vol. 31, no. 2pt4, pp. 845-854, May 2012. [Online]. Available: http://dx.doi.org/10.1111/j.1467-8659.2012.03065.x

[18] [T. F. Chen, G. V. G. Baranoski, B. W. Kimmel, and E. Miranda, "Hyperspectral modeling of skin appearance," ACM Trans. Graph., vol. 34, no. 3, pp. 31:1-31:14, May 2015. [Online]. Available: http://doi.acm.org/10.1145/2701416

[19] A. Sunguroff and D. Greenberg, "Computer generated images for medical applications," in Proceedings of the 5th annual conference on Computer graphics and interactive techniques, ser. SIGGRAPH'78. New York, NY, USA: ACM, 1978, pp. 196-202. [Online]. Available: http://doi.acm.org/10.1145/800248.807390

[20] H. Gouraud, "Computer display of curved surfaces," TC-20, IEEE, $\mathrm{p}$ 623,1971

[21] J. F. Blinn, "Models of light reflection for computer synthesized pictures," SIGGRAPH Comput. Graph., vol. 11, no. 2, pp. 192-198, Jul. 1977. [Online]. Available: http://doi.acm.org/10.1145/965141.563893

[22] C. Barillot, B. Gibaud, J.-m. Scarabin, and J.-1. Coatrieux, "3d reconstruction of cerebral blood vessels," IEEE Comput. Graph. Appl., vol. 5, no. 12, pp. 13-19, Dec. 1985. [Online]. Available: http://dx.doi.org/10.1109/MCG.1985.276258

[23] E. E. Catmull, "A subdivision algorithm for computer display of curved surfaces." Ph.D. dissertation, The University of Utah, 1974, aAI7504786.

[24] F. Neyret, R. Heiss, and F. Senegas, "Realistic rendering of an organ surface in real-time for laparoscopic surgery simulation," The Visual Computer, vol. 18, no. 3, pp. 135-149, 2002.

[25] J. F. Blinn and M. E. Newell, "Texture and reflection in computer generated images," Commun. ACM, vol. 19, no. 10, pp. 542-547, Oct. 1976. [Online]. Available: http://doi.acm.org/10.1145/360349.360353

[26] J. F. Blinn, "Simulation of wrinkled surfaces," SIGGRAPH Comput. Graph., vol. 12, no. 3, pp. 286-292, Aug. 1978. [Online]. Available: http://doi.acm.org/10.1145/965139.507101

[27] A. Hao, F. Song, S. Li, X. Liu, and X. Xu, "Real-time realistic rendering of tissue surface with mucous layer," in Computer Science and Engineering, 2009. WCSE '09. Second International Workshop on, vol. 1, Oct 2009, pp. 302-306.

[28] H. W. Jensen, S. R. Marschner, M. Levoy, and P. Hanrahan, "A practical model for subsurface light transport," in Proceedings of the 28th Annual Conference on Computer Graphics and Interactive Techniques, ser. SIGGRAPH '01. New York, NY, USA: ACM, 2001, pp. 511-518. [Online]. Available: http://doi.acm.org/10.1145/383259.383319

[29] M. A. ElHelw, S. Atkins, M. Nicolaou, A. Chung, and G.-Z Yang, Medical Image Computing and Computer-Assisted Intervention - MICCAI 2005: 8th International Conference, Palm Springs, CA, USA, October 26-29, 2005, Proceedings, Part I. Berlin, Heidelberg: Springer Berlin Heidelberg, 2005, ch. Photo-Realistic Tissue Reflectance Modelling for Minimally Invasive Surgical Simulation, pp. 868-875. [Online]. Available: http://dx.doi.org/10.1007/11566465_107

[30] Erik Ferguson, "Curious worm finite element organic tissue," http: //erikferguson.co.uk/The-Worm-series, 2014, accesso: 05/01/2016.

[31] M. Pharr and G. Humphreys, Physically Based Rendering, Second Edition: From Theory To Implementation, 2nd ed. San Francisco, CA, USA: Morgan Kaufmann Publishers Inc., 2010.

[32] O. Alexander, M. Rogers, W. Lambeth, J.-Y. Chiang, W.-C. Ma, C. C. Wang, and P. Debevec, "The digital emily project: Achieving a photorealistic digital actor," Computer Graphics and Applications, IEEE, vol. 30 , no. 4, pp. 20-31, July 2010.

[33] S. R. Marschner, S. H. Westin, E. P. F. Lafortune, K. E. Torrance, and D. P. Greenberg, "Image-based brdf measurement including human skin," in Proceedings of the 10th Eurographics conference on Rendering, ser. EGWR'99. Aire-la-Ville, Switzerland, Switzerland: Eurographics Association, 1999, pp. 131-144. [Online]. Available: http://dx.doi.org/10.2312/EGWR/EGWR99/131-144

[34] E. P. F. Lafortune, S.-C. Foo, K. E. Torrance, and D. P. Greenberg, "Non-linear approximation of reflectance functions," in Proceedings of the 24th annual conference on Computer graphics and interactive techniques, ser. SIGGRAPH '97.New York, NY, 
USA: ACM Press/Addison-Wesley Publishing Co., 1997, pp. 117126. [Online]. Available: http://dx.doi.org/10.1145/258734.258801

[35] A. Chung, F. Deligianni, P. Shah, A. Wells, and G.-Z. Yang, "Patient- specific bronchoscopy visualization through brdf estimation and disoc- clusion correction," Medical Imaging, IEEE Transactions on, vol. 25, no. 4, pp. 503-513, april 2006.

[36] L. ap Cenydd, N. John, M. Bloj, A. Walter, and N. Phillips, "Visualizing the surface of a living human brain," Computer Graphics and Applications, IEEE, vol. 32, no. 2, pp. 55-65, 2012.

[37] S. Yeung, H. Tsui, and A. Yim, "Global shape from shading for an endoscope image," in Medical Image Computing and ComputerAssisted Intervention - MICCAI'99, ser. Lecture Notes in Computer Science, C. Taylor and A. Colchester, Eds. Springer Berlin Heidelberg, 1999, vol. 1679, pp. 318-327.

[38] A. Malti and A. Bartoli, "Estimating the cook-torrance brdf parameters in-vivo from laparoscopic images," in Workshop on Augmented Environ- ment in Medical Image Computing and Computer Assisted Intervention (MICCAI), Nice, France, October/2012 2012.

[39] R. L. Cook and K. E. Torrance, "A reflectance model for computer graphics," ACM Trans. Graph., vol. 1, no. 1, pp. 7-24, Jan. 1982. [Online]. Available: http://doi.acm.org/10.1145/357290.357293

[40] A. Malti and A. Bartoli, "Combining conformal deformation and cooktorrance shading for 3-d reconstruction in laparoscopy," Biomedical Engineering, IEEE Transactions on, vol. 61, no. 6, pp. 1684-1692, June 2014

[41] M. Rump, R. Sarlette, and R. Klein, "Groundtruth data for multispectral bidirectional texture functions," in CGIV 2010. Society for Imaging Science and Technology, Jun. 2010, pp. 326-330

[42] P. Ren, J. Wang, J. Snyder, X. Tong, and B. Guo, "Pocke reflectometry," ACM Trans. Graph., vol. 30, no. 4, pp. 45:1-45:10, Jul. 2011. [Online].Available: http://doi.acm.org/10.1145/2010324.1964940

[43] M. Aittala, T. Weyrich, and J. Lehtinen, "Two-shot svbrdf capture for stationary materials," ACM Trans. Graph., vol. 34, no. 4, pp. 110:1110:13, Jul. 2015. [Online]. Available: http://doi.acm.org/10. $1145 / 2766967$
[44] A. Welch and M. van Gemert, Optical- Response of LaserIrradiated Tissue, ser. Lasers, Photonics, and Electro-Optics. Springer US, 1995. [Online]. Available: https://books.google.com.br/books?id= 11 w3wTVuAaoC

[45] P. Hanrahan and W. Krueger, "Reflection from layered surfaces due to subsurface scattering," in Proceedings of the 20th Annual Conference on Computer Graphics and Interactive Techniques, ser. SIGGRAPH '93. New York, NY, USA: ACM, 1993, pp. 165-174. [Online] Available: http://doi.acm.org/10.1145/166117.166139

[46] C. So-Ling and L. Li, "A multi-layered reflection model of natural human skin," in Computer Graphics International 2001. Proceedings, 2001, pp. 249-256

[47] W. L. Butler, "Absorption spectroscopy in vivo theory and application," Annual Review of Plant Physiology, vol. 15, no. 1, pp. 451-460, 1964

[Online]

Available: http://dx.doi.org/10.1146/annurev.pp.15.060164.002315

[48] P. Latimer, "A wave-optics effect which enhances light absorption by chlorophyll in vivo," Photochemistry and Photobiology, vol. 40, no. 2, pp. 193-199, 1984. [Online]. Available: http://dx.doi.org/10. 1111/j.1751-1097.1984.tb04575.x

[49] R. L. Olson, J. Gaylor, and M. Everett, "Skin color, melanin, and erythema," Archives of Dermatology, vol. 108, no. 4, pp. 541-544, 1973. [Online]. Available: +http://dx.doi.org/10.1001/archderm.1973. 01620250029008

[50] W. Matusik, H. Pfister, M. Brand, and L. McMillan, "A data-driven reflectance model," ACM Transactions on Graphics, vol. 22, no. 3, pp. 759-769, Jul. 2003

[51] W. I. M. Willaert, R. Aggarwal, I. Herzeele, N. J. Cheshire, and F. E. Vermassen, "Recent advancements in medical simulation: Patient-specific virtual reality simulation," World Journal of Surgery, vol. 36, no. 7, pp. 1703-1712, 2012. [Online]. Available: http: //dx.doi.org/10.1007/s00268-012-1489-0 